$R L-80-066$

\title{
Transient Stability of High Current Density Superconducting Wires
}

D E Baynham, VW Edwards and $M N$ Wilson

Paper presented at Applied Superconductivity Conference, Santa Fe, New Mexico, U S A. 29 September - 2. October 1980 
() The Science Research Council 1980

"The Science Research Council does not accept any responsibility

for loss or damage arising from the use of information contained in any of its reports or in any communication about its tests or investigations. 


\section{SUMMARY}

The transient stability of cooled superconductors is described by a theoretical model and the criteria for achieving an order of magnitude improvement in transient stability are delineated. Transient stability measurements on single wire $\mathrm{NoTi}$ and $\mathrm{Nb}_{3} \mathrm{Sn}$ sampies subjected to 100 usec heat pulses are reported. Samples cooled with a porous metal surface heat exchanger show the order of magnitude improvement in stability predicted by the theoretical model.

\section{INTRODUCTION}

The widespread application of superconductivity continues to be limited by magnet degradation and training. The effects are most marked in the straight sided magnets required for accelerator applications. In these medium size, high current density systems some level of degradation is usually present in that the magnet will only operate at a level below the basic conductor properties. However the more significant problem is magnet "training" with magnets often requiring many quencines to reach their final performance level. In a large accelerator with many hundreds of magnets, uncertain magnet behaviour represents a serious comissioning and operational problem.

The precise source of the quenching mechanism is not clear. In the absence of flux jumping, the most likely causes seem to be the sudden release of mechanical energy due to impregnant failure, conductor yielding and surface friction. On this premise progression to higher fields can only increase the training problem with the higher stress levels encountered. If training follows the square law of magnetic stress then the problem looks formidable for magnets designed to operate at 8-10 Tesla. We have therefore looked for a new approach to the problem of transient stability and training.

Considerable effort has already gone into the reduction of mechanical disturbances through the development of impregnation techniques, thermal contraction matching and the careful design of wagnet force supports. Although these precautions have produced some improvements, they have failed, in many instances, to reduce training to an acceptable level. This failure is perhaps not too surprising in view of the fact that, for an impregnated winding, the energy impulse needed to quench the magnet reduces to zero as the current approaches critical. Clearly the complete elimination of transient pulses in a highly stressed complex structure is unlikely. Faced with this problem the magnet designer must be conservative in defining operating level. An alternative approach is to accept the level of transient energy releases which seen to be occurring in present day magnets and develop conductors which can absotb such disturbances, with minimal temperature rise. At $4.2 \mathrm{~K}$ liquid helium offers by far the greatest potential as an energy absorber. This potential can only be realised for transient

disturbances if the heat transfer between conductor and liquid is significantly enhanced.

This paper describes the basic work which has been carried out to develop a conductor with an order of magnitude improvement in transient heat transfer characteristics giving a much improved transient stability. The criteria for transient stability are outlined with a relatively simple theoretical model.
Stability measurements on single wire $\mathrm{No}-\mathrm{Ti}$ and $\mathrm{Nb}_{3} \mathrm{Sn}$ samples are reported.

\section{TRANSIENT STABIIITY}

\section{General Considerations}

It is assumed that training is caused by the transient release of mechanical energy within the magnet winding. It has been postulated by Wipf ${ }^{\mathrm{J}}$ that the energy pulses form a generalised 'discurbance spectrum' ranging over both space and time. In the case of distributed transient disturbances covering a large volume there is essentially no conduction of heat to cold surroundings and the temperature rise is adiabatic. In contrast, for point disturbances, heat can diffuse away from the disturbance point and this aids stability. This paper concentrates on distributed transient disturbances since these represent the most severe instability problem. Basically, stabilisation against distributed transients relies on increasing the conductor unit heat capacity to a level where characteristic energy puises can be absorbed with minimal temperature rise. This increase in energy absorption capacity must be achieved with very little dilution of overall current density. Good thermal contact between the conductor and absorber is essential, otherwise thermal diffusion times will seriously reduce absorber efficiency.

Assuming that $10 \%$ of the winding volume might be available for energy absorption, Fig.(1) shows the potential of various absorbers in a "typical magnet". By comparing the lowest curve with the observed training behaviour of fully impregnated magnets in the medium size range, we may infer that the disturbances actually occuring in these magnets are $210^{3}-10^{4} \mathrm{~J} \mathrm{~m}^{-3}$. If such disturbances are to be tolerated when the conductor is working at critical current, it is clear from Fig.(1) that liquid helium is the only possible absorber.

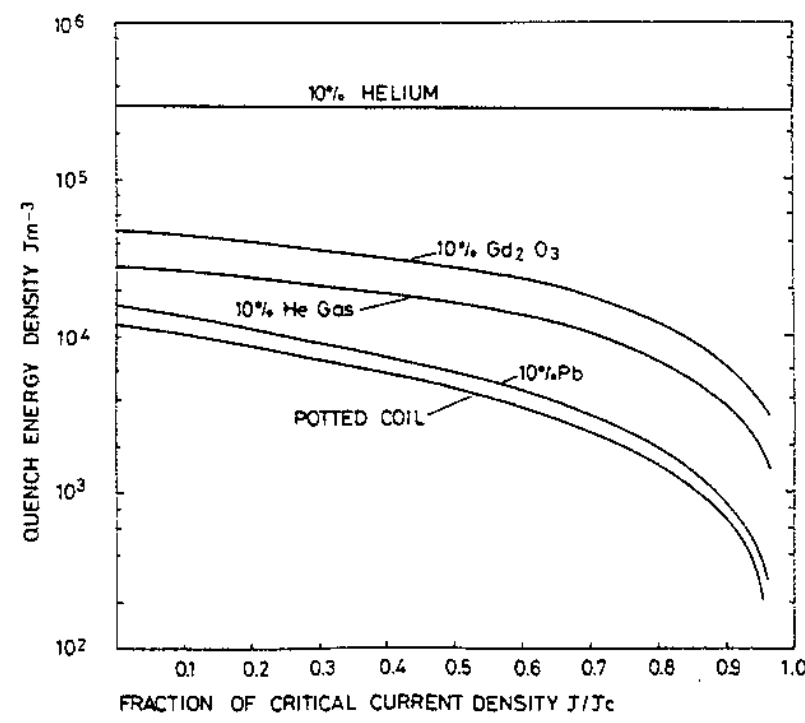

Fig. 1. Potential of energy absorbers in a "typical" magnet matrix at 6 Tesla. Matrix: $30 \% \mathrm{NbTi}, 40 \% \mathrm{Cu}, 30 \%$ epoxy. 
Transient Heat Transfer

Fig.(1) is based purely on heat capacity and takes no account of heat transfer capability or heat diffusion criteria. There is clearly no possibility of meeting steady state heat transfer criteria at the high levels of current density which are required. Measurements of transient heat transfer to liquid helium have been made by Schmidt ${ }^{2}$, Tsukamoto ${ }^{3}$ and Steward ${ }^{4}$. This work shows that much higher levels of heat transfer can be sustained under transient conditions; the transient heat transfer coefficient of $0.5 \times 10^{4} \mathrm{Wm}^{-1} \mathrm{~K}^{-1}$ is essentially constant until the onset of film boiling. Schmidt has formulated an approximate model for the. transient heat transfer mechanism. During the transient heating period, heat diffuses into the liquid adjacent to the conductor surface. The transition to film boiling occurs when sufficient energy has been absorbed to vaporise the helium in this diffusion layer. If the heating pulse is removed before the transition to film boiling then the system will recover. Therefore, for a given pulse length, the limiting energy which can be absorbed in a stable manner is given by the latent heat of the helium in the diffusion layer ie:

$$
q_{t}=\frac{\pi}{2} \gamma L\left(t_{p} D\right)^{\frac{1}{2}} J^{-2}
$$

where $h=$ latent heat of helium/unit volume

$t=$ pulse length; $D=$ diffusivisity of liquid helium. Fig.(2) is a comparison between this model and reported measurements. Although the agreement is adeouate to confirm the general basis of the Schmidt model, there is an appreciable discrepancy at short pulse times. We have therefore used the best fit line in Fig.(2) for stability calculations.

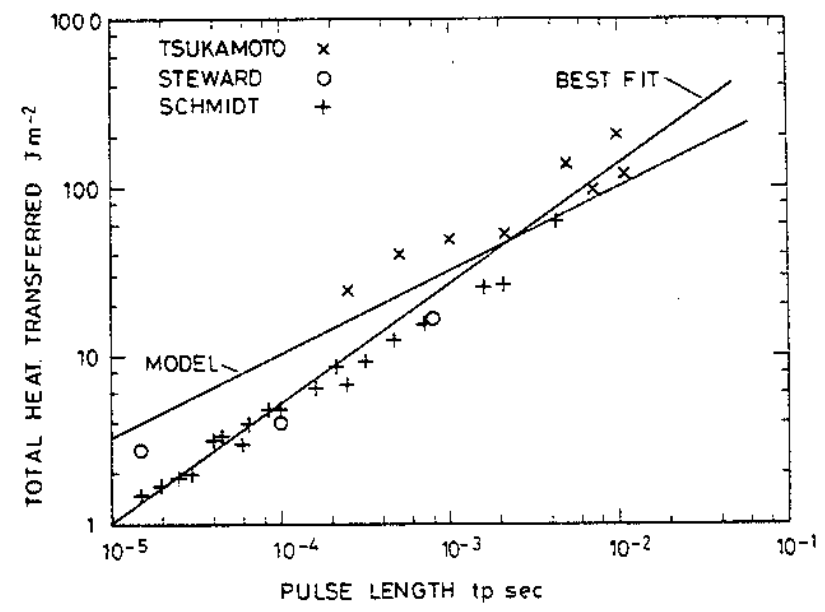

Fig. 2. Transient heat transfer to liquid helium.

\section{Stability Mode1}

This model of transient heat transfer has been used by $W_{i j} \operatorname{son}^{5}$ as the basis of a stability model for cooled conductors. The stability model represents a cooled conductor operating at critical current which is driven into the current sharing regime by a transient heat pulse. Considering only a distributed transient situation simplifies the model since longitudinal heat conduction can be ignored. Conductor stability ie. whether the conductor quenches or recovers depends on two main criteria. Firstly, it is necessary to satisfy the classical Stekly cryostability criterion; ie cooling exceeds heating. Secondly, it is necessary for the superconducting state to be recovered before the "transient allocation" of nucleate boiling heat transfer is exceeded. Two parameters are determined by the theoretical model, the total heat to be transferred to the liquid helium and the total pulse time.
For an input pulse with intrinsic energy density $Q_{d}$ $\mathrm{Jm}^{-3}$ the integrated heat energy which must be transferred is given by the sum of the original disturbance and subsequent Joule heating, which may be shown to be:

$$
q_{p}=\frac{A}{p} \frac{Q_{d}}{(j-\alpha)} \mathrm{Jm}^{-2}
$$

where $\mathrm{P} / \mathrm{A}=$ surface area/volume for the conductor $\alpha=$ Stekly parameter.

The period during which this heat must be transferred is $t_{p}=t_{d}+t_{r}$ where $t_{d}$ is the initial disturbance time and $t_{r}$ is the recovery time. Reference to Fig.(2) gives the limiting energy $q$. which can be transferred to the liquid in pulse time $t_{p}$. If $q_{p}<q_{t}$ then the conductor will recover, if $q_{p}>q_{t}{ }^{p}$ the conductor will quench. The exact value of $Q_{d}$ is found by iteration.

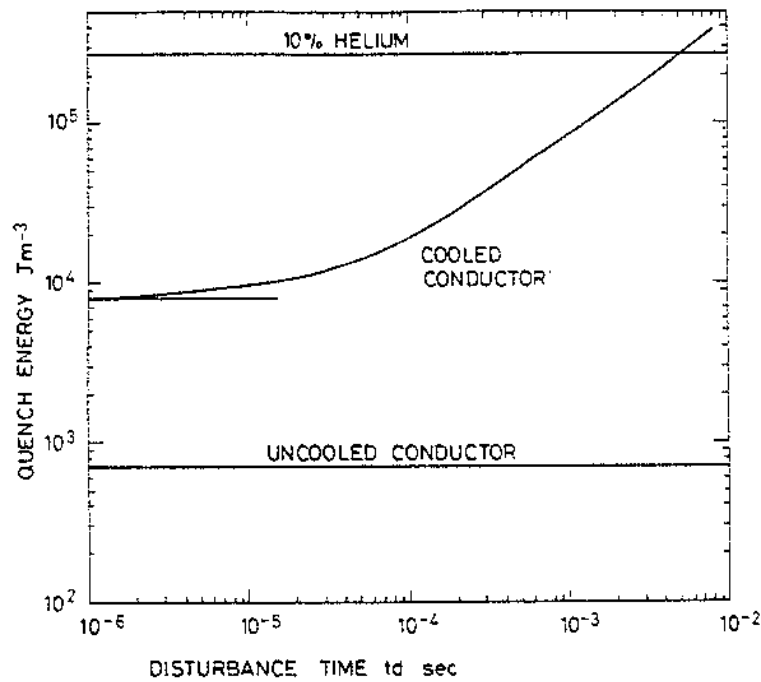

Fig. 3. Predicted quench energy for a NbTi conductor as a function of disturbance time. Magnetic field $B=5.5$ Iesla, conductor matrix Cu:NbTi, $2: 1$, diameter $=1 \mathrm{~mm}$.

Fig.(3) shows the calculated energy density to quench a conductor cooled by liquid helium and operating at critical current. The quench energy decreases with pulse length reaching a minimum value for an instantaneous pulse. The energy to quench the same conductor when uncooled and operating at $80 \%$ of critical current is also shown. Stability with helium cooling is improved by at least an order of magnitude. However, for a pulse of $100 \mu \mathrm{sec}$ duration the energy to quench the conductor is still only $1 \%$ of the latent heat of liquid helium. This is a direct result of the fact that heat can diffuse only $\sim_{3}$ wr into the liquid helium in $2100 \mu \mathrm{sec}$ and highlights the heat transfer problem. It is only for pulses of $210 \mathrm{msec}$ that $10 \%$ by volume of liquid can be used effectively. Thus, al though $10 \%$ of helium might be included in a winding matrix only a small fraction is effective in stabilisation against pulses in the microsecond regime.

Very little experimental evidence exists on the time scale of energy releases in magnet structures. Acoustic emission measurements on superconducting composites ${ }^{6}$ have shown that energy releases due to microplastic yielding have a duration $100 \mathrm{\mu sec}$. Crack propagation velocities for epoxy resins at low temperature are $210^{3} \mathrm{~m} \cdot \mathrm{sec}^{-1}$. Energy releases due to impregnant failure must consequently occur on the microsecond to millisecond time scale. A satisfactory stabilisation mechanism should therefore be effective for pulses in the microsecond range. This can only be achieved with liquid helium if the transient heat transfer characteristics are significantly improved. 
Heat transfer capability depends on the surface to volume ratio of the conductor. Some improvement might be produced by reducing the conductor diameter but to achieve an order of magnitude improvement would require an impractical conductor unit. The more practical solution, originally suggested by $\mathrm{W}$ A Steyert, is to use a porous metal conductor coating as a heat exchanger. Such a system combines high heat capacity, high surface area and relatively good thermal conductivity allowing very efficient heat exchange. With an order of magnitude increase in conductor surface area the full potential of liquid helium stability might be realised for pulses of a few microseconds duration. Porous Heat Exchangers

Porous metal structures can be formed by the sintering of fine metallic powders. This type of system already forms the basis of heat exchangers in low temperature refrigerators. Assuming spherical particles, a $30 \mathrm{\mu m}$ coating of $10 \mu \mathrm{m}$ spheres is sufficient to increase the surface area of our lmm conductor by an order of magnitude. The technique is therefore capable of weeting the spatial and surface area requirements for stabilising heat exchangers. We have studied the compatibility of sintered systems with superconductors. In particular sintering temperatures are important since this heat treatment must not degrade the superconauctor properties. Silver powder can be readily sintered by a heat treatment of approximately one hour at $300^{\circ} \mathrm{C}$. Some precompression of the powder is necessary to produce a satisfactory end product. This level of heat treatment is compatible with Nb-Ti conductors. Copper powder does not sinter at Low temperature and it is necessary to heat treat at $700^{\circ} \mathrm{C}$ to form a suitable matrix. This temperature precludes the use of copper powder with NbTi conductors but is compatible with reaction temperature for $\mathrm{Nb}_{3} \mathrm{Sn}$.

In order to test our stability predictions sintered heat exchangers were formed on single superconducting wires in a stainless steel channel mould. A micrograph of a typical system is shown in Fig.(4). The particle size of the silver powder varied from 50Nm to dust. It is seen that the heat exchanger occupies $220 \%$ by volume of the conductor unit. The metal density is $250 \%$ giving approximately $10 \%$ of helium by volume in the unit.

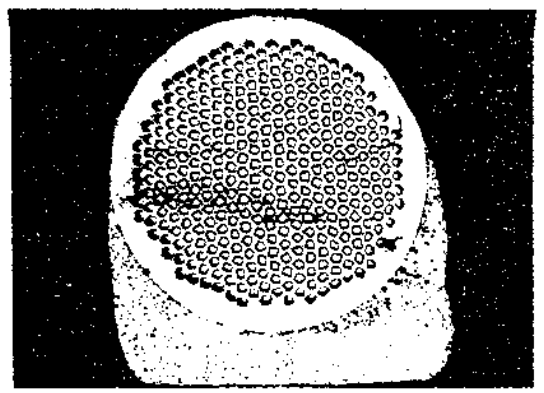

Fig. 4. NbTi conductor with sintered silver heat exchanger. Conductor diameter $=1$ mum.

\section{MEASUREMENT TECHNIQUE}

The measurement of transient conductor stability under cooled conditions revolves mainly around the problem of applying short heat pulses to the conductor. Indirect heater systems are unsuitable for a number of reasons. It is very difficult to estimate the proportion of heater energy transmitted to the conductor since heat is also lost to the coolant. Heat must also diffuse through an insulation layer and the heater generally impairs cooling. To be effective the heating mechanism must generate heat directly in the conductor

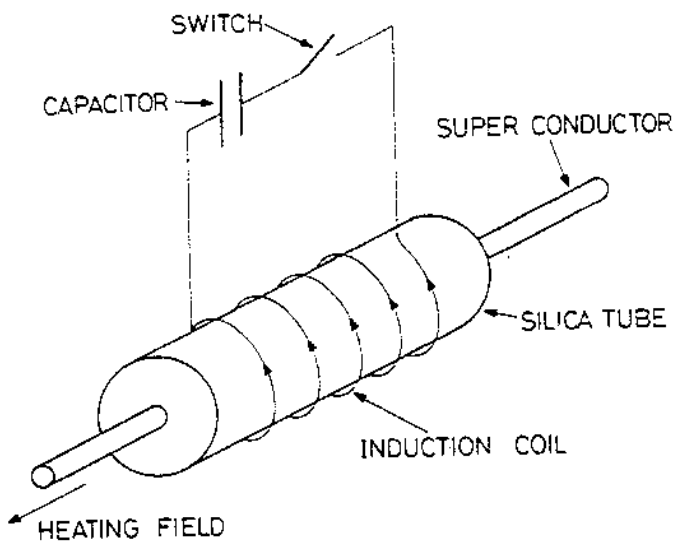

Fig.5. Transient induction heating technique. Basic parameters: $\mathrm{L}=24 \mu \mathrm{H}, \mathrm{C}=8 \mu \mathrm{F}, \mathrm{f}=11.5 \mathrm{kHz}, \tau=100 \mathrm{\mu sec}$.

leaving the surface fxee for heat transier. Direct heating is wost efficientiy achieved by eddy current or induction heating techniques. Conductors generally have an outer shell of copper which varies in thickness according to the design. Heating can be confined to this surface layer by applying a high frequency tagnetic field along the conductor axis. In this way energy is deposited uniformly around the conductor circumference and the inner filament region sees no electromagnetic disturbance. Diffusion of heat across the conductor occurs on a very short time scale, i-5 usec, depending on the matrix material.

Transient pulses of high frequency magnetic field were generated by the simple capacitor discharge system shown in Fig.(5). The conductor was inserted in a silica tube which carried the induction heating coil. The silica tube also provided thermal insulation between the wire and induction coil and accurately defined the helium volume surrounding the conductor. Energy was discharged into the induction coil with a simple switch. The system was designed to be series resonant at the desired frequency. With a high purity copper coil and leads, decay time constants for the heating pulse of up to $300 \mathrm{\mu sec}$ were achieved. Heater pulses were trimed by the addition of an external resistance wire. The energy deposited in the wire by the transient pulse may be shown to be:

$$
E=\frac{\mathrm{n}^{2} \mathrm{cCV} \mathrm{V}^{2} \tau}{\delta \mathrm{dL}} \mathrm{Jm}^{-3}
$$

where $\rho=$ wire resistivity.

$C=$ circuit capacitance $\quad \delta=$ skin depth

$=$ coil inductance

$\mathrm{V}=$ capacitor voltage

$\mathrm{n}=$ coil turns $/ \mathrm{m}$

$\tau=$ time constant

Induction coil transient currents were measured to verify the magnetic field expressions and to check that no appreciable loss occurred in the switch.

\section{STABILITY MEASÜREMENTS}

Conductor stability measurements were made with a heater length of $40 \mathrm{~mm}$ which was calculated to be sufficiently long for the simulation of distributed pulses. The complete stability sample was mounted in a swall superconducting solenoid with a transverse field of 5.5 Tesla. Measurements were made with heater pulse time constants of $100-120 \mu \mathrm{sec}$. These pulses were equivalent to square pulses of $50-60$ usec duration. In order to represent an uncooled conductor and confirm the energy scale, samples were also tested in vacuum. This was achieved by isolating the conductor at room temperature and allowing the cooldown to produce the 
vacuum conditions. Under vacuum, with no transverse heat transfer, the energy to quench the conductor should equal the enthalpy change to raise the conductor to its critical temperature.

Niobjum Titanium

Stability measurements on a standard lmm niobium titaniur conductor are presented in Fig.(6). The lower curve is the result of measurements with the sample in vacuum. The dashed. curve is the estimated enthalpy for the sample and is seen to be in good agreement with vacuum measurements, thus confirming the eddy current calculations. Enthalpy calculations were based on a critical current of Ic $=480$ amps which is the estimated critical current under well cooled conditions. Measurements with the sample in vacuum deviate from the enthalpy curve at high currents because of joule heating in the sample due to the resistive transition of the conductor. At very low currents the sample is able to recover by longitudinal conduction of heat from the sample ends. Measurements with the standard wire in liquid helium are shown by the middle curve. The measured quench energy at critical current is in very good agreement with the predictions of our theoretical model. The upper curve shows the stability of a wire with a heat exchanger of sintered silver powder such as that shown in Fig.(4). This sample was first tested in vacuum in order to establish the energy scale. With helium present the results indicate that the full heat capacity of a $10 \%$ volume fraction of liquid is being utilised for stabilisation.

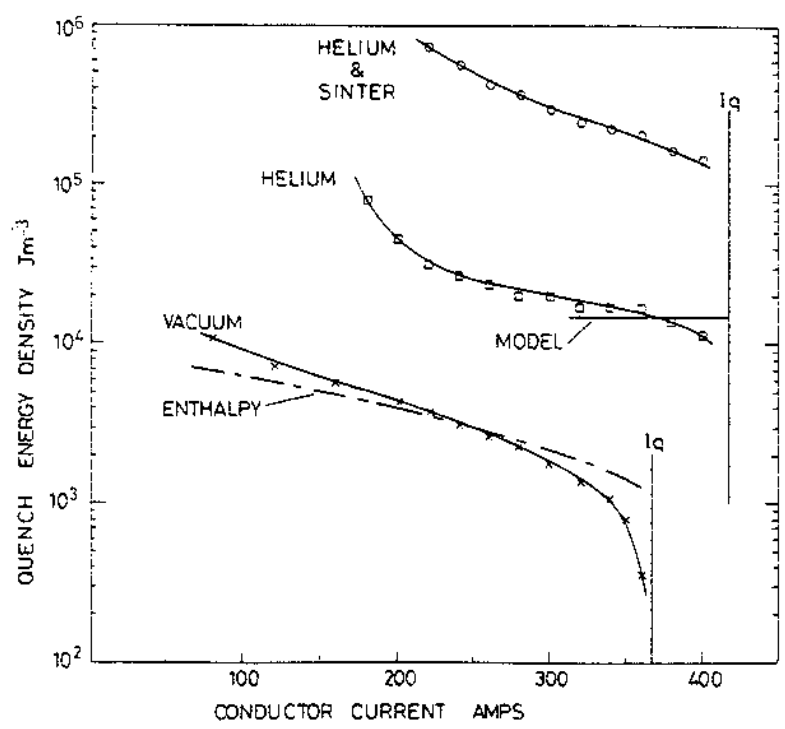

Fig.6. Quench energy density vs conductor current for $\mathrm{NbTi}$ conductor. Conductor matrix Cu:NbTi, 2:1, diameter=1 im, $B=5.5$ Tesla.

\section{Niobium Tin}

Fig.(7) shows the corresponding set of stability measirements for a multifilamentary $\mathrm{Nb}_{3} \mathrm{Sn}$ conductor. The conductor was of copper ring type construction with $65 \%$ copper in the outer shell protected by a diffusion barrier. Measurements in vacutm again show good agreement with conductor enthalpy; the divergence at low currents is more pronounced than in the case of $\mathrm{NbTi}$ but this might be expected because of the greater critical temperature. Measurements in liquid helium are in good agreement with the theoretical predictions. The enhanced stability provided by a porous silver heat exchanger is shown by the upper curve.

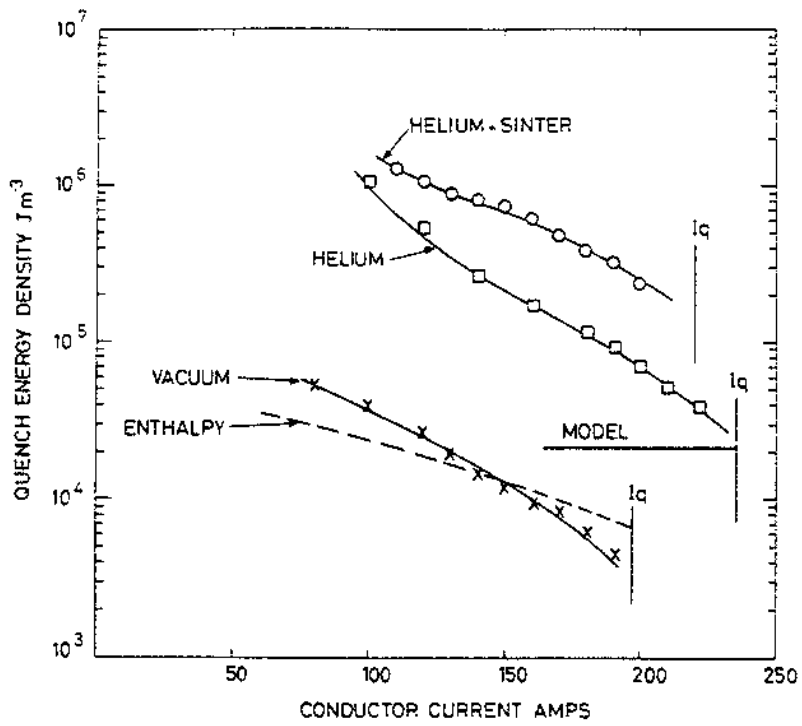

Fig.7. Quench energy density vs conductor current for $\mathrm{Nb}_{3} \mathrm{Sn}$ conductor. Conductor matrix Nb. Bronze $27 \%$, Cu. $65 \%$, Barrier $8 \%$, dianeter $=0.7 \mathrm{~mm}, \mathrm{~B}=5.5$ Tesla.

\section{CONCLUSTONS}

Measurements of transient conductor stability under cooled conditions have been made and are seen to be in good agreement with theoretical model calculations. A very significant improvement in stability for shor pulses, 100 usec, has been achieved by the use of porous metal heat exchangers. This improvement in stability has been realised within a volume equivalent to the voidage or solder volume normally found in braided or cabled conductors (15\%-25\%). Sinter filled, multj-strand conductors should therefore be stable against transient disturbances at the $10^{5} \mathrm{Jm}^{-3}$ level. This should give at least an order of magnitude safety margin over the transient disturbances which are believed to occur in present day magnets, $10^{3}-10^{4} \mathrm{Jm}^{-3}$. It therefore seems Iikely that magnets made with these stablised conductors will reach short sample current with absolutely no training.

\section{ACKNOWLEDGEMENTS}

We wish to acknowledge the contribution of $D$ Evans and $G$ hall in the preparation of sintered samples. Sample conductors were supplied by IMI Ltd and Airco Superconductors Ltd.

\section{REFERENCES}

1). Wipf, S.L. Los Alamos Report iA 7275 .

2). Schmidt, C. Appl.Phys.Lett. 32. 12 p827 (1978).

3). Tsukamoto, 0., Kobayashi, S. J. of App.Phys. Vol. 46. 3 p1359 (1975).

4) Stewaxd, W.G. Int. J. Heat and Mass Transfer 21 p863 (1978).

5) Wilson, M.N. Rutherford Laboratory Internal Note SMR $/ 39$.

6). Schmidt, C, Pasztor, G. IEEE Trans on Magnetics MAG-13, 116, 1977 . 